\title{
Protective effects of molecular hydrogen on hepatotoxicity induced by sub-chronic exposure to chlorpyrifos in rats
}

\author{
Zhi-ming Xun ${ }^{1, A, D \oplus}$, Fei Xie ${ }^{2, A-B \oplus}$, Peng-xiang Zhao ${ }^{2, C \oplus}$, Meng-yu Liu' ${ }^{2, E}{ }^{\oplus}$, Zhi-yuan Li, ${ }^{1, B \oplus}$, \\ Jian-mei Song ${ }^{1, B \oplus}$, Xian-min Kong ${ }^{3, E \oplus}$, Xue-mei Ma ${ }^{2, A} \oplus$, Xiao-yang Li ${ }^{1, A, F \oplus}$ \\ ${ }^{1}$ Chao-Yang Hospital, Capital Medical University, Beijing, China \\ ${ }^{2}$ College of Life Science and Bioengineering, University of Technology, Beijing, China \\ ${ }^{3}$ Commission for Science and Technology, Miyun District, Beijing, China \\ A - Research concept and design, B - Collection and/or assembly of data, C - Data analysis and interpretation, \\ $D$ - Writing the article, $E$ - Critical revision of the article, $F$ - Final approval of article
}

Zhi-ming Xun, Fei Xie, Peng-xiang Zhao, Meng-yu Liu, Zhi-yuan Li, Jian-mei Song, Xian-min Kong, Xue-mei Ma, Xiao-yang Li. Protective effects of molecular hydrogen on hepatotoxicity induced by sub-chronic exposure to chlorpyrifos in rats. Ann Agric Environ Med. $2020 ; 27$ (3): 368-373. doi: $10.26444 /$ aaem/125504

\begin{abstract}
Introduction. Chlorpyrifos (CPF) is a organophosphate insecticide widely used in agriculture with attendant adverse health outcomes. Chronic exposure to CPF induces oxidative stress and elicits harmful effects, including hepatic dysfunction. Molecular hydrogen has been identified as a novel antioxidant which could selectively scavenge hydroxyl radicals.

Objective. The aim of this study was to determine whether the intake of hydrogen-rich water (HRW) could protect rats from hepatotoxicity caused by sub-chronic exposure to CPF.

Materials and method. Rats were treated with hydrogen-rich water by oral intake for 8 weeks. Biochemical indicators of liver function, SOD and CAT activity, GSH and MDA levels were determined by the spectrophotometric method. Liver cell damage induced by CPF was evaluated by histopathological and electron microscopy analysis. PCR array analysis was performed to investigated the effects of molecular hydrogen on the regulation of oxidative stress related genes.

Results. Both the hepatic function tests and histopathological analysis showed that the liver damage induced by CPF could be ameliorated by HRW intake. HRW intake also attenuated CPF induced oxidative stress, as evidenced by restored SOD activities and MDA levels. The results of PCR Array identified 12 oxidative stress-related genes differentially expressed after CPF exposure, 8 of chich, including the mitochondrial Sod2 gene, were significantly attenuated by HRW intake. The electron microscopy results indicated that the mitochondrial damage caused by CPF was alleviated after HRW treatment. Conclusions. The results obtained suggest that HRW intake can protect rats from CPF induced hepatotoxicity, and the oxidative stress signaling and the mitochondrial pathway may be involved in the protection of molecular hydrogen.
\end{abstract}

\section{Key words}

oxidative stress, hepatotoxicity, chlorpyrifos, molecular hydrogen, mitochondrial pathway

\section{INTRODUCTION}

Organophosphorus pesticides (OPs) are widely used to control insect pests in households and agriculture. OPs can cause dysfunction of the nervous system by inhibiting the activity of acetylcholinesterase (AChE). Due to the acute high-level exposure of OPs, they are likely to cause severe a severe 'cholinergic syndrome' in humans and other mammals [1], but chronic low-level exposure has been implicated as a serious risk to human health [2]. Chlorpyrifos (CPF) is a widely used, broad spectrum and moderately toxic OPs used in households and agriculture, despite the United States Environmental Protection Agency imposing restrictions in 2000 on the use of CPF on agricultural crops. CPF itself has less activity to inhibit $\mathrm{AChE}$ and requires metabolic activation to attain its potential toxic form, CPF-oxon (CPO), by CYP450-mediated oxidative desulfuration, primarily in the liver [3].

Cholinesterase inhibition is the most important mechanism involved in CPF induced toxicity. However, there

Address for correspondence: Xiao-yang Li, Chao-Yang Hospital, Capital Medical University, Beijing, China

E-mail:Vxy_li@163.com

Received: 24.03.2020; accepted: 16.07.2020; first published: 11.08.2020 are reports that oxidative stress also plays an important role in the mechanism of inducing CPF toxicity. CPF can induce oxidative stress in organs such as the brain and liver by increasing the level of reactive oxygen species (ROS) [4, 5]. Oxidative stress can induce organ toxicity; therefore, supplementation of antioxidants is considered an alternative method for reducing the toxicity of CPF [5]. With the increased use of CPF in agricultural production, readily available and effective antioxidants have great potential for improving CPF induced toxicity.

In 2007, Ohsawa et al. discovered that molecular hydrogen exerts a therapeutic antioxidant activity by selectively neutralizing hydroxyl radicals, without interfering with other physiological ROS [6]. Subsequent studies have shown that molecular hydrogen has widely therapeutic effects on various diseases by reducing oxidative stress, inflammation and apoptosis, including ischemia-reperfusion injury, atherosclerosis, type 2 diabetes, metabolic syndrome, and Parkinson's disease [7-11]. These clinical studies have shown that molecular hydrogen has great potential and can be used as an effective preventive and therapeutic antioxidant.

Our previous study has demonstrated that oral intake of hydrogen-rich water (HRW) could play a protective role in the neurotoxicity induced by chronic exposure to a sub- 
threshold dose of CPF [12]. Since the liver is the organ where activation and detoxification of CPF takes place, whether HRW has protective effects on the CPF induced hepatic toxicity remains unknown.

\section{OBJECTIVE}

The study has two aims: to identify the potential role of HRW on the hepatotoxicity caused by sub-chronic exposure to CPF, and to investigate the mechanisms underlying the protective effects of molecular hydrogen.

\section{MATERIALS AND METHOD}

Chemicals and preparation of hydrogen water. Technical grade CPF ( $>98 \%$, Sigma-Aldrich, USA) was prepared by corn oil to obtain an effective concentration of $6.75 \mathrm{mg} / \mathrm{kg}$ body weight (b.w.). Ultra-pure water was used throughout the experiment for preparation of the reagents and buffers used for various biochemical assays in the study. The hydrogenrich water was produced by placing a metallic magnesium stick (Friendear, Tokyo, Japan) into drinking water $\left(\mathrm{Mg}+2 \mathrm{H}_{2} \mathrm{O} \rightarrow \mathrm{Mg}(\mathrm{OH})_{2}+\mathrm{H}_{2}\right.$; final hydrogen concentration: $0.55-0.65 \mathrm{mM} ; \mathrm{pH} 8.0)$. The magnesium stick contained 99.9\% pure metallic magnesium in a natural stone and polypropylene ceramic container. HRW was stored under atmospheric pressure at $4^{\circ}$ in an aluminum bag without dead volume.

Animals and experimental design. This study was performed on male Wistar albino rats weighing 120-150 g, housed under standard conditions at $22^{\circ} \mathrm{C}-25^{\circ} \mathrm{C}$ with a $12 \mathrm{~h}$ light-dark cycle and on a normal diet. Before the experiments, the animals had one week to adapt to laboratory conditions.

Thirty rats were divided at random into three groups, each group consisting of 10 animals. Rats in the control group (C/oil) served as the control and were given only corn oil ( $2 \mathrm{ml} / \mathrm{kg}$ b.w.) and normal water. Rats in the CPF group were administered CPF (1/20 LD $\mathrm{LD}_{50} \sim 6.75 \mathrm{mg} / \mathrm{kg}$ b.w., $\left.1 \mathrm{ml}\right)$ once daily by oral gavage, and drank normal water. In the HRW group, CPF-intoxicated rats were given HRW by oral intake for $1 \mathrm{~h}$ each time, twice a day. The regimens were performed once daily and lasted eight weeks. At the end of the experiment, rats were euthanized using isofluorane anaesthesia. The blood of each rat was collected by cardiac puncture. Liver and brain tissues were carefully excised, washed with ice-cold physiologic saline $(0.9 \%, \mathrm{w} / \mathrm{v})$, and fixed in $4 \%$ paraformaldehyde for histopathological examination, or stored at $-80^{\circ}$ in aliquots for oxidative stress biomarkers measurement and RNA extraction.

All experimental protocols were approved by the Animal Care and Use Committee of the Capital Medical University (ACUCCMU No. 1002032) and were performed in accordance with the Regulations for the Administration of Affairs Concerning Experimental Animals (China).

Biochemical Indicator of Liver Function. Plasma aspartate aminotransferase (AST), alanine aminotransferase (ALT) and alkaline phosphatase (ALP) activities were determined spectrophotometrically using commercial diagnostics kits (Randox, United Kingdom).
Histopathological and ultrastructural analysis. For histological analysis, samples were cut into pieces and fixed with $4 \%$ paraformaldehyde in $0.1 \mathrm{M}$ phosphate buffer, and then embedded in paraffin wax, mounted onto slides, and stained with haematoxylin and eosin (H\&E). Mounted slides were examined and photographed under an Olympus light microscope.

Electron microscopy analysis. The ultra-thin sections were cut at $0.1 \mu \mathrm{m}$ and stained with uranyl acetate and lead citrate. A Hitachi H-7650 transmission electron microscope was used to capture images.

SOD, CAT, GSH and MDA asssays. Assay kits were used according to the manufacture's protocol to measure the SOD activity (Dojindo, Japan). CAT activity was measured by the Goth method and expressed as $\mathrm{mU} / \mathrm{mg}$ protein by the rate of decreased hydrogen peroxide [13]. GSH was measured according to the recycling system by reaction with DTNB and GSH (Dojindo, Japan). Absorbance was quantified at $405 \mathrm{~nm}$. The concentration of GSH in sample solutions was determined using a calibration curve and expressed as nmol of GSH per mg protein. A thiobarbituric acid reactive species (TBARS) assay kit was used to measure MDA levels (Cayman, USA). Taking BSA as the standard, the total protein concentration was determined by the Bradford Assay (Tiangen, China). All results were expressed as $[\mathrm{MDA}] \mathrm{in} \mathrm{nmol} / \mathrm{mg}$ of protein.

Real-time microarray and real-time PCR analysis. RNA from samples was extracted with a RNeasy Microkit (Qiagen). Total RNA concentration for each sample was first determined by 260:280 nm absorbance ratio, after which equal amounts of RNA were reverse transcribed to form cDNA. For real-time microarray analysis, samples were screened for the expression of 84 key genes from the oxidative stress pathway by using the $\mathrm{RT}^{2}$ Profiler PCR array plate (Superarray Biosciences) on a Stratagene Mx3005P detector. Raw data from the realtime microarray analysis was uploaded using a PCR array data analysis template available at (http://www.sabiosciences. $\mathrm{com} / \mathrm{pcr} /$ arrayanalysis.php). A two-fold or greater change in expression with $\mathrm{P}<0.05$ was considered significant. Real-time PCR analysis was performed using a Maxima SYBR Green qPCR Master Mix (Fermentas, EU). The PCR primer sequences were obtained from qPrimerDepot (http://primerdepot.nci. nih.gov/) and synthesized by Shanghai Sangon (Shanghai, China). This used GAPDH as the reference gene and the data was analyzed by the comparative threshold cycle $(\mathrm{Ct})$ method.

Statistical Analysis. All data represent the results from at least three independent experiments and expressed as the mean \pm standard error of the mean (SEM). Statistical analysis of the data was performed through one-way analysis of variance (ANOVA). A 2-tailed probability level of $\mathrm{P}<0.05$ was used to indicate statistical significance. Non-significant results are expressed as ns.

\section{RESULTS}

Effects of CPF and HRW treatment on liver enzymes activities. The extent of hepatic injury was assessed by the release into a plasma of the liver enzymes including ALT, AST and ALP. Compared with the control group, eight weeks of 


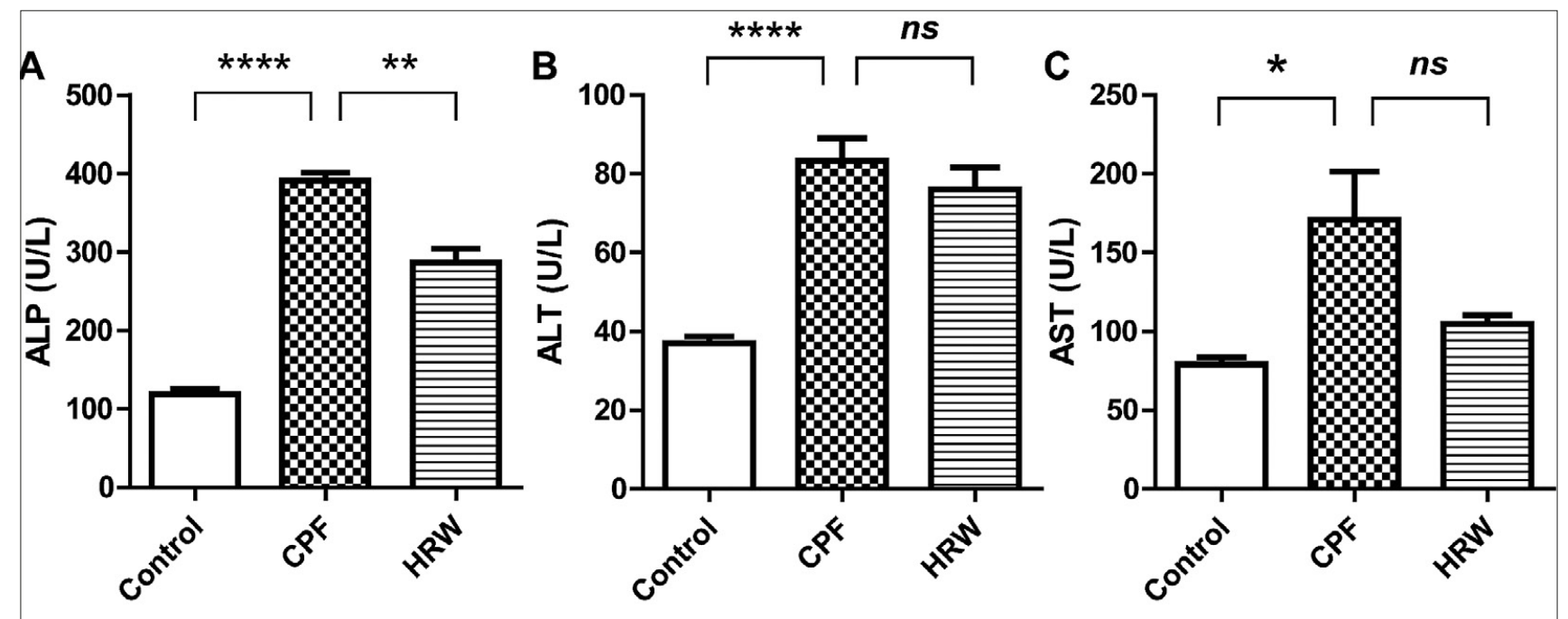

Figure 1. Effects of CPF exposure on the liver enzymes activities and the protective effect of HRW. The results are expressed as the means $\pm \mathrm{SEM}$. ${ }^{*} \mathrm{P}<0.05,{ }^{* *} \mathrm{P}<0.01$, $* * * * P<0.0001$

CPF intake caused a significant increase in serum enzymes ALT (125.23\%; $\mathrm{P}<0.0001)$, AST (116.24\%; $\mathrm{P}=0.0143)$, and ALP (229.49\%; $\mathrm{P}<0.0001)$ (Fig. 1). After HRW treatment, the activity of ALP was markedly decreased $(26.75 \% ; \mathrm{P}=0.0011)$ when compared with the CPF group. The activity of AST in the HRW group was reduced (38.77\%) with marginal significance $(\mathrm{P}=0.0858)$ relative to the $\mathrm{CPF}$ group. Only a slight decrease in ALT activity $(8.60 \%$; $\mathrm{P} \geq 0.05)$ was observed in the HRW group compared with the CPF group.

Histopathological changes. Liver in the control group showed regular structured hepatic parenchyma with hepatocytes around the central veins and sinusoid capillaries exhibiting normal endothelial cells. After CPF treatment, histopathological analysis revealed slight hepatic injury, evidenced by hepatocyte swelling and focal piecemeal necrosis. These histopathological changes were not obvious, however, in the HRW group (Fig. 2A).

A detailed analysis of the alterations caused by CPF and HRW treatment was performed by electron microsopy. After $\mathrm{CPF}$ treatment, it was found that hepatocyte mitochondria had significant structural changes, swelling, increased matrix space and amorphous matrix density. In the HRW group, most mitochondria remained intact, with a dark matrix and relatively regular distribution, and the degree of swelling reduced (Fig. 2B).

HRW intake can effectively reduce liver oxidative stress caused by CPF. Oxidative stress indicators, including SOD and CAT activity and levels of MDA and GSH of the liver

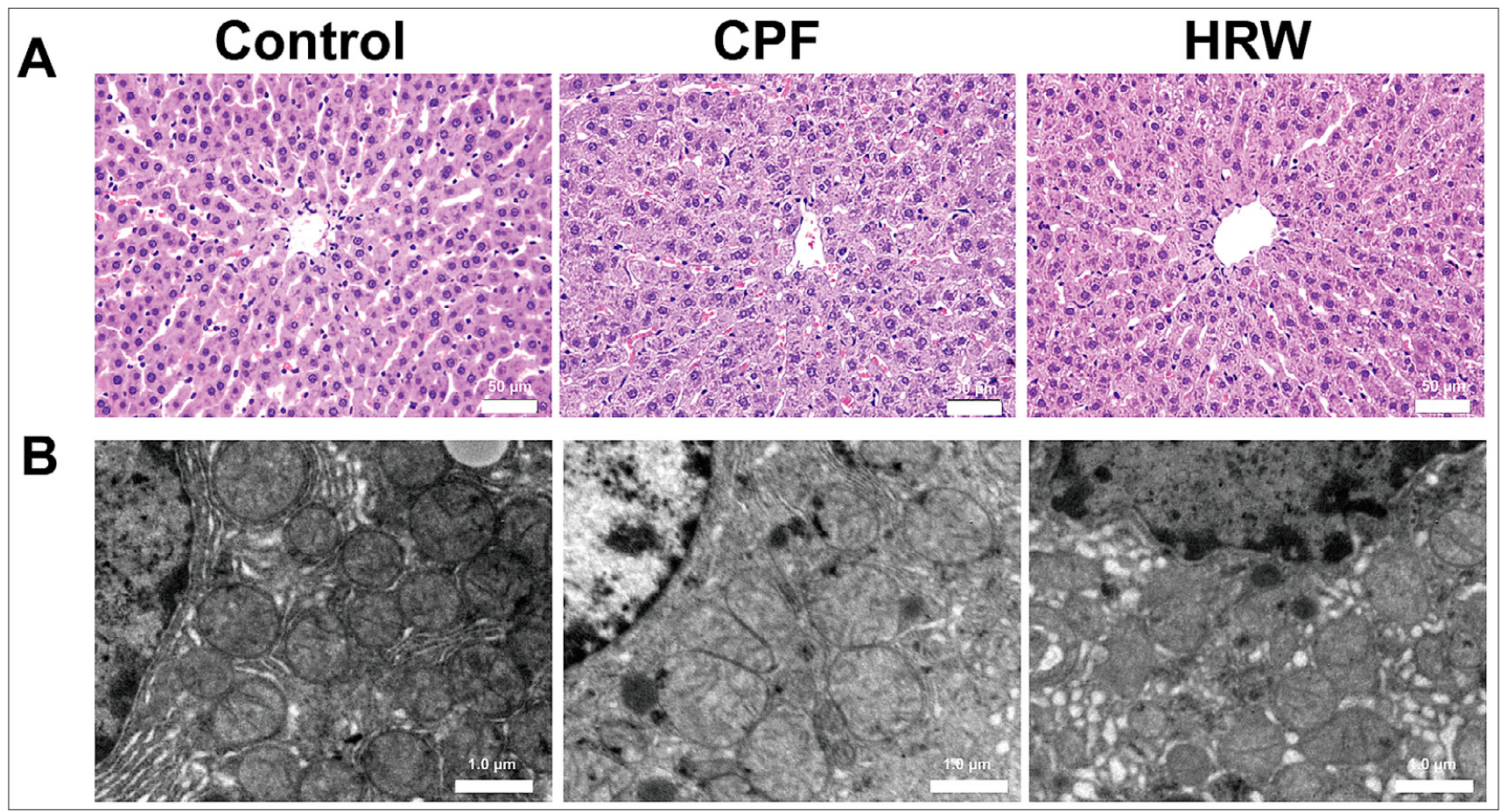

Figure 2. Representative histological and ultrastructural changes in the liver after treatment with CPF or HRW. (A) CPF induced changes in rat liver histology and the protective effects of HRW. (B) HRW intake alleviated CPF induced ultrastructural changes in mitochondria 
tissues, were determined after eight weeks of $\mathrm{CPF}$ intake. $\mathrm{CPF}$ treatment resulted in a significant decrease $(28.38 \% ; \mathrm{P}=0.0163)$ in the hepatic enzyme activity of SOD, and the effect was significantly reversed by HRW treatment $(39.78 \% ; \mathrm{P}=0.0153)$ compared to the CPF group (Fig. 3A). The activity of CAT was also reduced $(24.10 \%)$ after CPF treatment, which was reduced by the daily intake of HRW (13.37\%); these changes, however, were without statistical significance ( $\mathrm{P} \geq 0.05)$ (Fig. 3B).

MDA as a biomarker of the free radical-mediated oxidation process and its concentration results showed that CPF exposure of eight weeks can lead to markedly increased liver MDA levels (51.17\%; $\mathrm{P}=0.0102)$, and the intake of HRW can significantly decrease MDA levels (34.60\%; $\mathrm{P}=0.0388$ ), compared to the CPF group (Fig. 3C). Both CPF and HRW treatment had no significant effect on the GSH levels in the liver (Fig. 3D).

Effects on gene expression profile of oxidative stress-related genes. The study used real-time PCR-based microarray to compare the expression changes of 84 genes in signaling pathways related to oxidative stress. Five of the 84 genes (5.95\%) were upregulated after treatment with $\mathrm{CPF}$, only the expression of Scd1 (63.20\%; P=0.0059) and Sod $2(71.15 \%, \mathrm{P}=0.0018)$ were significantly ameliorated by HRW treatmen (Fig. 4A). CPF treatment also downregulated the expression of seven genes, six of which were markedly reversed by HRW treatment, including Cat (48.09\%; P < 0.0001), Gclc (40.78\%; P=0.0466), Gclm (65.43\%; P=0.0020), Gpx1 (47.89\%; P=0.0066), Idh1 (27.30\%; $\mathrm{P}=0.0005)$ and Nox4 (72.97\%; $\mathrm{P}=0.0355)$ (Fig. 4B).

Differential regulation of Sod 2 expression by CPF in liver and brain. The expression of Sod 2 after CPF and HRW treatment in the liver and the brain was compared by realtime PCR using Taqman probes. The results showed that the expression of Sod2 in the liver was increased by 3.51fold after treatment with CPF, and the elevated expression was significantly attenuated $(65.48 \% ; \mathrm{P}=0.0007)$ by $\mathrm{HRW}$ treatment, which was consistent with the PCR array results. However, neither the CPF or HRW treatment significantly changed Sod 2 expression in the brain tissues (Fig. 4C).

\section{DISCUSSION}

According to a report, the average daily intake of CPF is about $0.005 \mathrm{ug} / \mathrm{kg} /$ day for an adult weighing $70 \mathrm{~kg}$ [14]. Most laboratory animal long-term toxicology studies are conducted by the oral route, usually at least two orders of magnitude higher than the dose during occupational exposure, with a dose range of $0.05-25 \mathrm{mg} / \mathrm{kg} /$ day [14]. It is notable that the CPF in the current study was at a subthreshold dose level equivalent to $1 / 20$ acute oral LD50 (6.75 mg/kg/day).

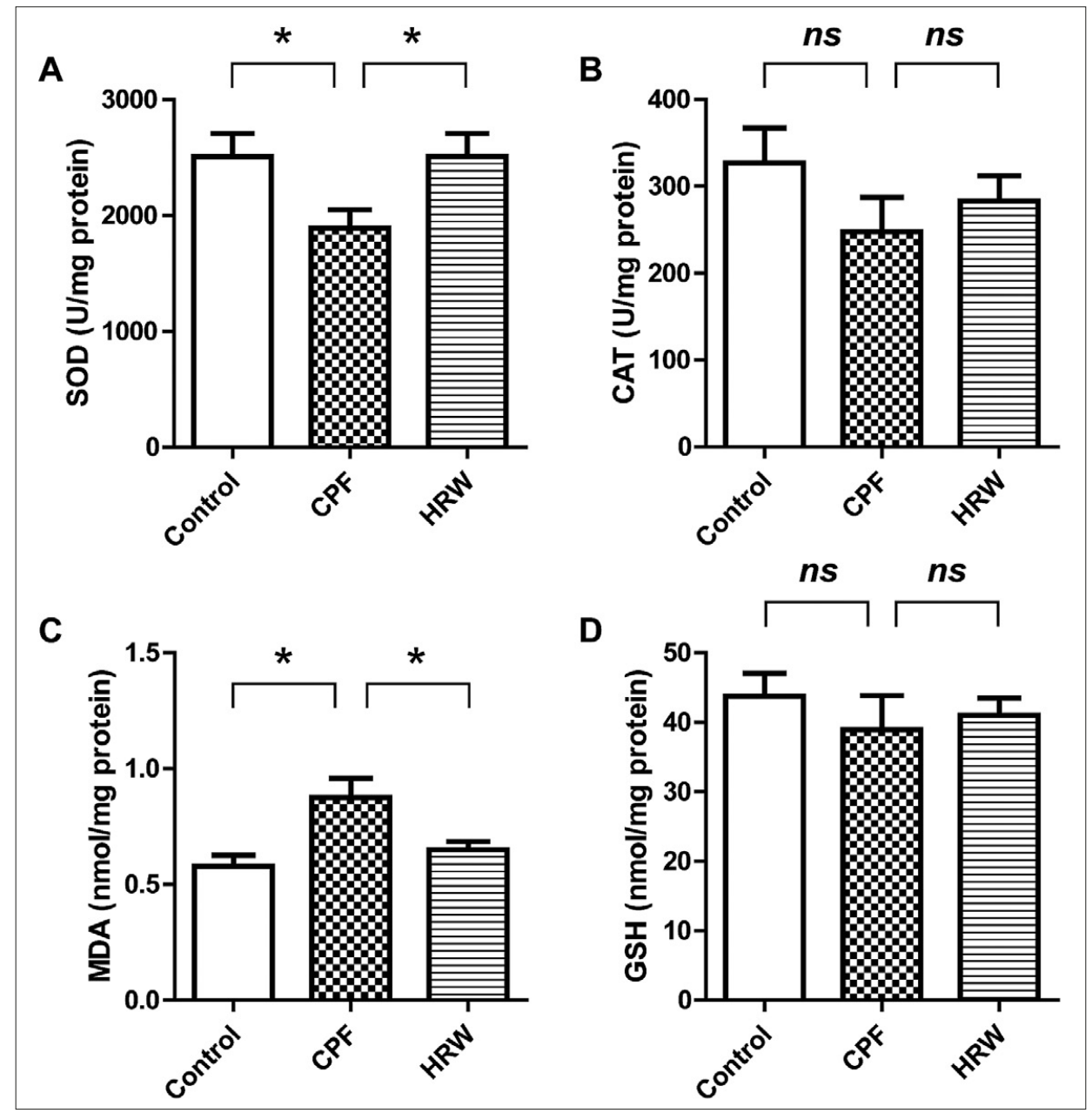

Figure 3. HRW intake attenuated CPF induced oxidative stress in the liver of rats. Oxidative stress markers, including (A) SOD, (B) CAT, (C) MDA, (D) GSH, were determined to assess the CPF induced oxidative stress. The results are expressed as the means \pm SEM. ${ }^{*} \mathrm{P}<0.05$ 


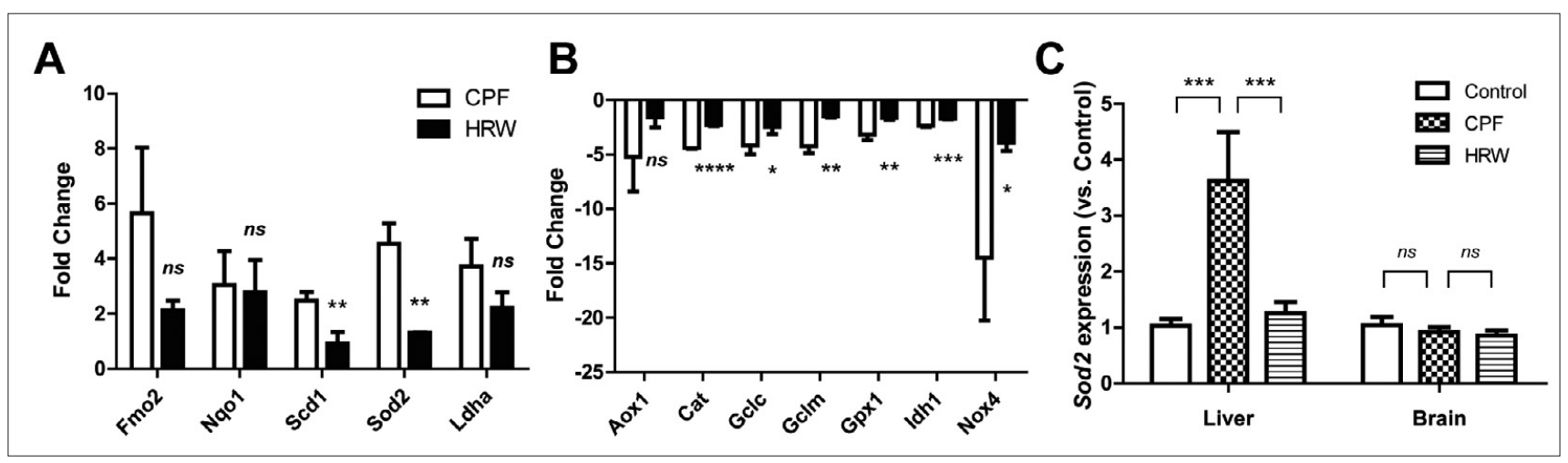

Figure 4. Fold change difference in mRNA expression of genes regulating oxidative stress in the liver of rats after CPF or HRW treatment. (A) PCR array results showing genes that were significantly upregulated by CPF exposure. (B) Genes that were markedly downregulated by CPF exposure. (C) Sod2 expression was differentially regulated by CPF exposure in the liver and the brain. The results are expressed as the means $\pm S E M$. ${ }^{*} P<0.05$, ${ }^{* *} P<0.01,{ }^{* * *} P<0.001,{ }^{* * * *} P<0.0001$.

Due to the important role of the liver in the detoxification of heterologous organisms, it is particularly susceptible to damage caused by environmentally heterogeneous organisms. Plasma enzymes (including ALT, AST and ALP) are the most sensitive biomarkers, and liver toxicity damage is positively correlated with its concentration. This study shows that $\mathrm{CPF}$ intake can significantly increase the concentration of ALT, AST and ALP in rat plasma, which is consistent with previous reports $[15,16]$. The increase in liver enzymes activities may be caused by liver dysfunction and disturbance in the biosynthesis of these enzymes, with alteration in the permeability of the liver membrane, which allows them to escape from the liver cytosol into the blood circulation [17]. Experiments have shown that the concentration of enzymes in the blood of rats ingesting HRW is reduced to varying degrees, indicating that HRW protects the toxic damage of liver cells through a mechanism that is not fully understood, maintains the integrity of the membrane, and limits the leakage. Hepatic function tests confirmed the histopathological lesions observed in the present study. CPF exposure resulted in degenerative changes in hepatocyte and congestion in the central veins of the liver, and this damage could be ameliorated by HRW intake.

Oxidative stress is a balance between the degree of oxidative stress and antioxidant activity. Pesticides are known to induce oxidative stress, and extensive studies suggest that production of the reactive oxygen species (ROS) can be a major contributor to the toxicity of pesticides [18]. SOD, as a defence against toxic effects of ROS, metabolizes superoxide radicals to hydrogen peroxide and oxygen.

The current research revealed that CPF exposure significantly decreased SOD activities in the liver of rats. The results were in agreement with a previous study [16]. CAT helps in the removal of $\mathrm{H}_{2} \mathrm{O}_{2}$ formed during the reaction catalyzed by SOD. Only a slight decline in CAT activity by CPF exposure was observed, and the reduction in CAT activity following low doses of CPF exposure has also been reported in a previous study [19]. GSH, synthesized primarily in the hepatic cells, plays a key role in cellular antioxidant defences by scavenging reactive oxygen species and reducing lipid peroxides. No significant changes in GSH levels were recorded after CPF treatment, which indicated the insensitivity of GSH level to low-level of CPF exposure. As the final product of membrane lipid peroxidation, MDA has been widely used as a marker of the degree of oxidative damage to cells. In the current study, the content of MDA in rat liver tissues treated with CPF was significantly increased, indicating that the peroxidation of liver cell membranes increased, and the change in membrane permeability led to the leakage of enzymes in liver cells. The use of Serum AST, ALT, increased the ALP enzyme. After the HRW treatment, the oxidative stress status in the liver was improved, SOD activities and MDA levels were restored, CAT activities and GSH levels were also slightly increased, all of which may be due to the attenuated liver damage.

Rat oxidative stress $\mathrm{RT}^{2}$ Profiler PCR Array is used to comprehensively evaluate changes in the oxidative stress pathway in CPF and determine whether there is a new pathogenic mechanism. This array involves the expression of the 84 genes of oxidative stress. Twelve differentially expressed genes were identified that clearly distinguish the CPF group from the control group, including five upregulated genes and seven down-regulated genes. Among the 12 differentially expressed genes, eight were significantly attenuated by HRW treatment, which indicated involvement of the oxidative stress pathway in the protection of molecular hydrogen against the $\mathrm{CPF}$ induced hepatic toxicity. In future research, all these differentially expressed genes should be studied in greater depth.

In this study, great attention was paid to the Sod2 gene that encodes a mitochondrial protein that forms a homotetramer, and binds one manganese ion per subunit. SOD2, as a part of the iron/manganese superoxide dismutase family, is responsible for superoxide detoxification in mitochondria [20]. Expression of the Sod 2 gene in boththe liver and brain of rats was determined. The results showed that CPF exposure significantly enhanced the Sod 2 expression in liver, an effect that was markedly attenuated by HRW treatment. However, the significant alteration in Sod2 expression after CPF or HRW treatment was not observed in the brain. As the major site for detoxification or biotransformation of xenobiotics, the liver may be the final target site of CPF, which may be responsible for the different changes in Sod2 expression between the liver and the brain.

Furthermore, it is worth noting that the results of the ultrastructural analysis indicated the potential involvement ofa mitochondrial mediator in the protection of molecular hydrogen from CPF induced hepatic toxicity. After HRW treatment, the mitochondrial damage caused by CPF is reduced. The alteration in the expression of mitochondrial Sod 2 gene caused by CPF or HRW treatment, may also indicate the potential involvement of a mitochondrial 
mediator. Since molecular hydrogen is a highly diffusible gas, it can penetrate biomembranes smoothly to diffuse into the mitochondria. Several previous studies also provided evidence of the protective role of molecular hydrogen on the mitochondria. Cui et al. reported that molecular hydrogen significantly attenuated mitochondrial permeability transition pore (mPTP) opening in cerebral I/R animals during reperfusion [21]. Another study demonstrated that molecular hydrogen also reduces infarct size in a canine model of cardiac ischemia-reperfusion injury, via the opening of the mitochondrial $\mathrm{K}_{\text {ATP }}$ channels, followed by inhibition of mitochondrial permeability transition pores [22]. Therefore, the detailed mechanism involved in the protective effect of molecular hydrogen on mitochondria needs further study.

Exposure to pesticides has additionally been shown to be highly correlated with respiratory pathologies (asthma, chronic obstructive pulmonary disease (COPD) and lung cancer) $[23,24]$. Previous studies have demonstrated that molecular hydrogen could exert protective effects on various respiratory symptoms, such as asthma [25], COPD [26, 27, $28]$, and lung cancer $[29,30]$, suggesting that molecular hydrogen may also have protective effects on respiratory system injury caused by pesticides exposure, although this, too, need to be further investigated.

\section{CONCLUSION}

The presented study has demonstrated that ingestion of HRW can protect Wistar rat liver from CPF induced toxic damage, and its protective mechanism seems to be mediated by the molecular hydrogen antioxidant activity. The study also provided evidence indicating the potential involvement ofa mitochondrial mediator in the protective role of molecular hydrogen. Therefore, these findings indicate that the potential feasibility of ingesting HRW can protect people from hepatotoxic damage caused by OPs exposure in daily production and life.

\section{REFERENCES}

1. Costa LG. Current issues in organophosphate toxicology. Clinica Chimica Acta. 2006; 366(1-2): 1-13.

2. Murray A, Rathbone AJ, Ray DE. Novel protein targets for organophosphorus pesticides in rat brain. Environ Toxicol Pharmacol. 2005; 19(3): 451-454.

3. Betancourt AM, Carr R L. The effect of chlorpyrifos and chlorpyrifosoxon on brain cholinesterase, muscarinic receptor binding, and neurotrophin levels in rats following early postnatal exposure. Toxicoll Sci. 2004; 77(1): 63-71.

4. Mehta A, Verma RS, Srivas N. Chlorpyrifos induced alterations in the levels of hydrogen peroxide, nitrate and nitrite in rat brain and liver. Pesticide Biochem Physiol. 2009; 94(2-3): 55-59.

5. Uchendu C, Ambali SF, Ayo JO. The organophosphate, chlorpyrifos, oxidative stress and the role of some antioxidants: a review. African Agric Res. 2012; 7(18): 2720-2728

6. Ohsawa I, Ishikawa M, Takahashi K, Watanabe M, Nishimaki K, et al. Hydrogen acts as a therapeutic antioxidant by selectively reducing cytotoxic oxygen radicals. Nature Med. 2007; 13(6): 688-694.

7. Fukuda KI, Asoh S, Ishikawa M, Yamamoto Y, Ohsawa I, Ohta S. Inhalation of hydrogen gas suppresses hepatic injury caused by ischemia/reperfusion through reducing oxidative stress. Biochem Biophysic Res Communic. 2007; 361(3): 670-674.

8. Ohsawa I, Nishimaki K, Yamagata K, Ishikawa M, Ohta S. Consumption of hydrogen water prevents atherosclerosis in apolipoprotein E knockout mice. Biochem Biophysic Res Communic. 2008; 377(4): 1195-1198.
9. Kajiyama S, Hasegawa G, Asano M, Hosoda H, Fukui M, Nakamura $\mathrm{N}$, et al. Supplementation of hydrogen-rich water improves lipid and glucose metabolism in patients with type 2 diabetes or impaired glucose tolerance. Nutr Res. 2008; 28(3): 137-143.

10. Nakao A, Toyoda Y, Sharma P, Evans M, Guthrie N. Effectiveness of hydrogen rich water on antioxidant status of subjects with potential metabolic syndrome-an open label pilot study. J Clin Biochem Nutr. 2010; 46(2): 140-149.

11. Fu Y, Ito M, Fujita Y, et al. Molecular hydrogen is protective against 6-hydroxydopamine-induced nigrostriatal degeneration in a rat model of Parkinson's disease. Neurosci Lett. 2009; 453(2): 81-85.

12. Wang TT, Zhao L, Liu MY, Xie F, Mei XM, et al. Oral intake of hydrogenrich water ameliorated chlorpyrifos-induced neurotoxicity in rats. Toxicol Applied Pharmacol. 2014; 280(1): 169-176.

13. Goth L. A simple method for determination of serum catalase activity and revision of reference range. Clinica Chimica Acta 1991; 196(2-3): 143-151.

14. Eaton DL, Daroff RB, Autrup H, Bridges J, Buffler P, Costa LG, et al. Review of the toxicology of chlorpyrifos with an emphasis on human exposure and neuro-development. Critical Rev Toxicol. 2008; 38(1): $1-125$.

15. Mansour SA, Mossa ATH. Oxidative damage, biochemical and histopathological alterations in rats exposed to chlorpyrifos and the antioxidant role of zinc. Pesticide Biochem Physiol. 2010; 96(1): 14-23.

16. Raina R, Baba NA, Verma PK, Sultana M, Singh M. Hepatotoxicity induced by subchronic exposure of fluoride and chlorpyrifos in wistar rats: mitigating effect of ascorbic acid. Biol Trace Element Res. 2015; 166(2): 157-162.

17. Khan SM, Sobti RC, Kataria L. Pesticide-induced alteration in mice hepato-oxidative status and protective effects of black tea extract. Clinica Chimica Acta. 2005; 358(1-2): 131-138.

18. Abdollahi M, Ranjbar A, Shadnia S, Nikfar S, Rezaie A. Pesticides and oxidative stress: a review. Med Sci Monitor. 2004; 10(6): 141-147.

19. Bebe FN, Panemangalore M. Exposure to low doses of endosulfan and chlorpyrifos modifies endogenous antioxidants in tissues of rats. J Environ Sci Health Part B 2003; 38(3): 349-363.

20. Zelko IN, Mariani TJ, Folz RJ. Superoxide dismutase multigene family: a comparison of the CuZn-SOD (SOD1), Mn-SOD (SOD2), and ECSOD (SOD3) gene structures, evolution, and expression. Free Radical Biol Med. 2002; 33(3): 337-349.

21. Cui Y, Zhang H, Ji M, Jia M, et al. Hydrogen-rich saline attenuates neuronal ischemia-reperfusion injury by protecting mitochondrial function in rats. J Surg Res. 2014; 192(2): 564-572.

22. Yoshida A, Asanuma H, Sasaki H, Sanda S, Yamazaki S, Asano Y, et al. $\mathrm{H}(2)$ mediates cardioprotection via involvements of $\mathrm{k}(\mathrm{atp})$ channels and permeability transition pores of mitochondria in dogs. Cardiovasc Drugs Ther. $2012 ; 26(3): 217-226$.

23. Tarmure S, Alexescu TG, Orasan O, Negrean V, Sitar-Taut AV, Coste $\mathrm{SC}$, et al. Influence of pesticides on respiratory pathology-a literature review. Ann Agric Environ Med. 2020; 27(2): 194-200.

24. Sak ZHA, Kurtuluş Ş, Ocakli B, Töreyin ZN, Bayhan İ, Yeşilnacar MI, et al. Respiratory symptoms and pulmonary functions before and after pesticide application in cotton farming. Ann Agric Environ Med. 2018; 25(4): 701-707.

25. Huang P, Wei S, Huang W, Wu P, Chen S, Tao A, et al. Hydrogen gas inhalation enhances alveolar macrophage phagocytosis in an ovalbumin-induced asthma model. Int Immunopharmacol. 2019; 74(105646): 1-10.

26. Liu X, Ma C, Wang X, Wang W, Li Z, Wang X, et al. Hydrogen coadministration slows the development of COPD-like lung disease in a cigarette smoke-induced rat model. Int J Chron Obstruct Pulmon Dis. 2017; 12: 1309-1324.

27. Liu Z, Geng W, Jiang C, Zhao S, Liu Y, Zhang Y, et al. Hydrogen-rich saline inhibits tobacco smoke-induced chronic obstructive pulmonary disease by alleviating airway inflammation and mucus hypersecretion in rats. Exp Biol Med. 2017; 242(15): 1534-1541.

28. Lu W, Li D, Hu J, Mei H, Shu J, Long Z, et al. Hydrogen gas inhalation protects against cigarette smoke-induced COPD development in mice. J Thorac Dis. 2018; 10(6): 3232-3243.

29. Wang D, Wang L, Zhang Y, Zhao Y, Chen G. Hydrogen gas inhibits lung cancer progression through targeting SMC3. Biomed Pharmacother. 2018; 104: 788-797.

30. Chen J, Kong X, Mu F, Lu T, Lu Y, Xu K. Hydrogen therapy can be used to control tumor progression and alleviate the adverse events of medications in patients with advanced non-small cell lung cancer. Med Gas Res. 2020; 10(2): 75-80. 